\title{
Erratum
}

\section{ESO imaging survey: optical deep public survey}

A. Mignano ${ }^{1,2,3}$, J.-M. Miralles ${ }^{2,4}$, L. da Costa 2,5 , L. F. Olsen ${ }^{6,2,7}$, I. Prandoni ${ }^{3}$, S. Arnouts ${ }^{8}$, C. Benoist ${ }^{6}$, R. Madejsky ${ }^{9}$, R. Slijkhuis ${ }^{2}$, and S. Zaggia ${ }^{10}$

\footnotetext{
1 Dipartimento di Astronomia, Universita' di Bologna, via Ranzani 1, 40126 Bologna, Italy e-mail: amignano@ira.inaf.it

2 European Southern Observatory, Karl-Schwarzschild-Str. 2, 85748 Garching b. München, Germany

3 INAF - Istituto di Radioastronomia, via Gobetti 101, 40129 Bologna, Italy

4 Tècniques d'Avantguarda, Avda. Carlemany 75, 700 Les Escaldes, Andorra

5 Observatorio Nacional, Rua Gel. Jose Cristino 77, Rio de Janeiro, RJ, Brazil

6 Observatoire de la Côte d'Azur, Laboratoire Cassiopée, BP4229, 06304 Nice Cedex 4, France

7 Dark Cosmology Centre, Niels Bohr Institute, University of Copenhagen, Juliane Maries Vej 30, 2100 Copenhagen, Denmark

8 Laboratoire d'astrophysique de Marseille, Traverse du Siphon, BP 8, 13376 Marseille Cedex 12, France

9 Universidade Estadual de Feira de Santana, Campus Universitário, Feira de Santana, BA, Brazil

10 Osservatorio Astronomico di Trieste, via GB Tiepolo 11, 34131 Trieste, Italy
}

A\&A 462, 553-563 (2007), DOI: 10.1051/0004-6361:20065030

Key words. catalogs - surveys - stars: general - Galaxy: general - radio continuum: general - errata, addenda

The third footnote on page 553 indicates an incorrect link to the CDS. Please read instead:

Available at CDS via anonymous ftp to cdsarc.u-strasbg.fr (130.79.128.5) or via

http://cdsweb.u-strasbg.fr/cgi-bin/qcat?]/A+A/462/553 